\title{
Model Reactions for Electrophilic Substitution of Aromatic Compounds
}

By Franz Effenberger ${ }^{[*]}$

Symmetrical triaminobenzenes are particularly suitable as model compounds for studying electrophilic aromatic reactions; due to the additive electron releasing influence of three mutually meta dialkylamino groups in (1), cationic intermediates are stabilized to such a degree that individual steps of electrophilic substitution can be separately studied. The stabilizing effect increases in the order morpholino $<$ piperidino $<$ dimethylamino $<$ pyrrolidino. By HMO calculations, the mesomeric interaction of the individual amino groups with the

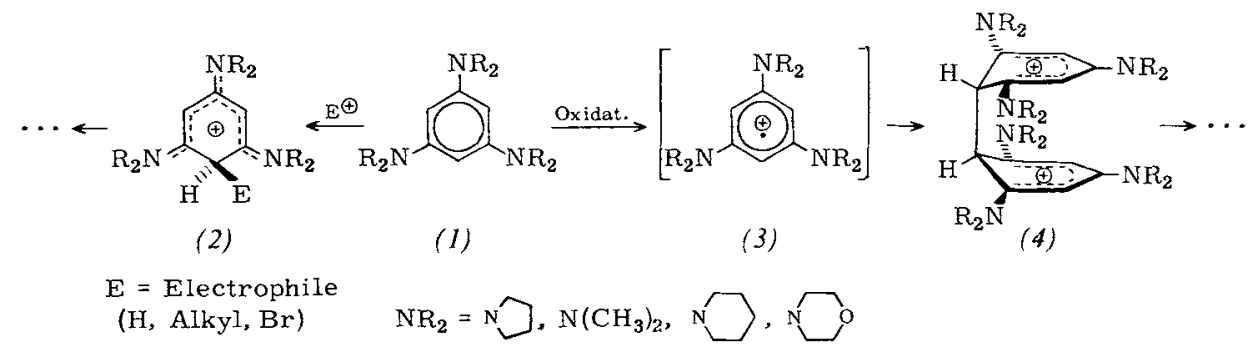

aromatic $\pi$ system may be assessed quantitatively from the position of the ${ }^{1} \mathrm{H}-\mathrm{NMR}$ ring proton signals if anisotropic effects are carefully eliminated.

The $\sigma$ complexes (2) formed on protonation, alkylation, and bromination of tripyrrolidinobenzene prove to be isolable; in some cases, primary reaction at a dialkylamino nitrogen of ( $I$ ) and subsequent rearrangement to (2) can be established. In contrast to the intermediates described by Olah, compounds (2) are true "Wheland intermediates", i.e. cationic analogs of the Meisenheimer complexes formed in the course of nucleophilic aromatic substitution.

Tris(dialkylamino)benzenes (I) may be oxidized readily; the isolable intermediates (4) are presumably formed by dimerization of (3). Spectra and model calculations offer unequivocal evidence for the "endo" conformation of (4); the preorientation thus indicated for dimerization of $(3)$ is significant also with regard to the benzidine rearrangement.

[*] Prof. Dr. F. Effenberger

Institut für Organische Chemie der Universität

7 Stuttgart, Azenbergstrasse 14 (Germany) 
In many cases the information gained from reactions of $(I)$ can be successfully applied to the reactions of $N, N$-dialkylanilines. Although isolation of cationic intermediates is not feasible here under normal conditions, the productdetermining steps may be influenced in a clearly defined way, based on the model concepts developed for $(l)$. This principle is exemplified by the action of bromine on $N, N$-dialkylanilines and the reversibility of bromination of dialkylanilines.

Lecture at Tübingen on June 25, 1971 [VB 322 IE] German version: Angew. Chem. 84, 37 (1972) 